\title{
Deactivation of Poly(o-Aminophenol) Film Electrodes After their Interac- tion with Ferric Cations. A Study Applying Interfacial Resistance Measu- rements
}

\author{
D. Scolari and R. Tucceri*
}

\begin{abstract}
Instituto de Investigaciones Fisicoquímicas Teóricas y Aplicadas (INIFTA), CONICET, Facultad de Ciencias Exactas, Universidad Nacional de La Plata, Sucursal 4, Casilla de Correo 16, (1900) La Plata, Argentina
\end{abstract}

\begin{abstract}
The experimental arrangement in this investigation is one in which poly $(o$-aminophenol) (POAP) films were supported on thin gold films. Freshly prepared POAP film electrodes were firstly deactivated by interaction with a ferric cation solution and then, they were reactivated by treatment with an alkaline solution. Cyclic Voltammetry (CV) and Surface Resistance (SR) measurements were performed to analyze if after consecutive deactivation and reactivation processes, the redox sites distribution (distance between adjacent redox sites) of POAP remained unaltered. By comparing with the resistance response of a freshly prepared POAP film, an attenuation of the gold film resistance within the potential region of maximal electroactivity of POAP was observed after polymer deactivation. This attenuated resistance change was attributed to the creation of polymer inactive zones at the gold film-POAP film interface. Thus, deactivated POAP films exhibit a redox sites distribution with larger distances between active redox sites, as compared with that present at a gold film surface contacting a non-deactivated poly (o-aminophenol) film. Surface Resistance also showed that while the redox sites distribution of a POAP film with a low degree of deactivation $\left(\theta_{\mathrm{c}}<0.4\right)$, can be recovered without distortion, the redox sites distribution of a POAP film with a degree of deactivation higher than 0.4 , suffers a irreversible distortion, which remains even after reactivation by alkaline treatment. This work can be interesting from the practical viewpoint, because conducting properties of POAP, which are associated to the electron hopping process between adjacent redox sites, should remain substantially unchanged before and after the polymer has been used. In this regard, the results of this work show the restrictive stability conditions of POAP.
\end{abstract}

Keywords: Poly(o-aminophenol) film electrodes, surface resistance, deactivation and reactivation processes.

\section{INTRODUCTION}

After redox transformation an electroactive polymeric material could exhibit different chemical and physical properties as compared from the reduced one. Combinations of different techniques, such as, cyclic voltammetry, spectroscopies and conductivity measurements, have supplied valuable information about the ionic and electronic charge transfer and transport processes in these materials [1-3]. These last techniques have contributed to the establishment of the chemical reaction mechanism during the redox process of some polymer materials. Voltammetric response at redox polymers in the background electrolyte solution (without monomer) represents in most cases, a combination of broad anodic and cathodic peaks with a plateau of the current at higher potentials. However, this simple result is a consequence of a complicated phenomenon which includes the faradaic process (generation of charged electronic entities at the polymer chains near the electrode surface by electron transfer to the metal), the transport of species throughout the film and the ion exchange at the film-solution interface (to keep the overall electroneutrality). Poly (o-aminophenol) (POAP), which is a ladder polymer [4-6], exhibits a one-step

*Address correspondence to this author at the Instituto de Investigaciones Fisicoquímicas Teóricas y Aplicadas (INIFTA), Sucursal 4, Casilla de Correo 16, (1900) La Plata, Argentina; Fax: (54) (0221) 425-4642;

E-mail: rtucce@inifta.unlp.edu.ar redox process [6]. Parameters characterizing charge-transfer and charge-transport processes at POAP films have been estimated using different techniques [4]. Barbero et al. [6] have studied the thermodynamic properties of a POAP film by cyclic voltammetry. Ohsaka et al. [7] studied the redox kinetics of a POAP-coated electrode by potential-step chronoamperometry and normal pulse voltammetry. Tucceri et al. [8] have studied spectroelectrochemical properties of POAP in the presence of different electrolytes and a redox mechanism was proposed in terms of a chemical reaction involving amine-imine sites. Also, the charge transport process at POAP films has been studied extensively by $a c$ impedance measurements [4]. Transport process across POAP films in the presence of redox active couples has been studied using rotating disc electrode voltammetry [9]. It is noteworthy that most basic results about POAP reviewed in the literature refer to conduction properties of POAP films with maximal electroactivity (freshly prepared). However, there is only a small number of papers about conduction properties of POAP films that have lost part of their optimum electroactivity (deactivated POAP films) [4]. Considering the growing applications of POAP [10], where the polymer is subjected to rugged conditions (chemical environment, potential range, prolonged experiments, storage conditions, etc.) that could cause a decay of the electrochemical activity, not many efforts have been made to study POAP deactivation. In this regard, in practical applications it is important that the reversible redox response and conducting 
properties of POAP films remain substantially unchanged before and after the films have been used. In previous works [11,12] spectroelectrochemical measurements and rotating disk electrode voltammetry (RDEV) were employed to study the deactivation of POAP films after their interaction with a ferric cation solution. The deactivation process of POAP by contact with a ferric cation solution was chosen because it was demonstrated by Cyclic Voltammetry [13] that the initially deactivated polymer can be partially reactivated by treatment with a $0.1 \mathrm{M} \mathrm{NH}_{4} \mathrm{OH}$ solution. In the present work, the Surface Resistance (SR) technique [14] was employed to study deactivation of POAP. SR is a non-traditional technique in electrochemistry, which is based in the electron dispersion process at scattering centres [14]. The experimental arrangement in this investigation is one in which a POAP film is supported on a thin gold film. By employing a thin gold film as base electrode one can apply simultaneously both Cyclic Voltammetry (CV), to characterize quantitatively the redox species transformation on the gold film surface contacting the polymer film, and Surface Resistance measurements to investigate changes in the electronic properties at the gold-POAP interface (distribution of scatterers) during the redox conversion of the polymer. Then, the aim of the present work was to deactivate POAP and then study how its redox sites distribution is modified with the degree of deactivation. In the context of the SR technique, redox sites of the polymer play the role of scattering centres for the conduction electrons of the base metal. The present work can help to obtain further information about the effect of the charge-propagation process on the practical applications of POAP.

\section{EXPERIMENTAL}

Eight gold thin film electrodes of constant thickness $\phi_{\mathrm{m}} \sim$ $30 \mathrm{~nm}$ were prepared by vacuum evaporation as was previously described $[8,14]$. These electrodes exhibit initial resistance values about $20 \mathrm{ohms}$. The relationship between the length $l$ and the width $w$ of these gold film electrodes was 2 . The electrode area was $1 \mathrm{~cm}^{2}$. POAP films were grown on these base electrodes following the procedure described in $[5,6]$. That is, polymer films were obtained by immersing the base electrodes in a $10^{-3} \mathrm{M}$ orthoaminophenol $+0.4 \mathrm{M} \mathrm{Na}$ $\mathrm{ClO}_{4}+0.1 \mathrm{M} \mathrm{HClO}_{4}$ solution and cycling the potential between -0.25 and $0.8 \mathrm{~V}$ (versus $\mathrm{SCE}$ ) at a scan rate $v=0.05$ $\mathrm{V} \mathrm{s}^{-1}$. These POAP-coated electrodes were then rinsed and transferred to the supporting electrolyte solution $(0.4 \mathrm{M} \mathrm{Na}$ $\mathrm{ClO}_{4}+0.1 \mathrm{M} \mathrm{HClO}_{4}$ ) free of monomer. Then, the voltammetric reduction charge $Q_{\text {Red,T }}$ in the latter solution was determined by integration of the cathodic current of the voltammetric response between $-0.2 \mathrm{~V}$ and $0.5 \mathrm{~V}$ (versus SCE) $\left(v=0.01 \mathrm{~V} \mathrm{~s}^{-1}\right)$. In the same way as in [15], POAP films were grown up to an approximate thickness of $\phi_{\mathrm{p}} \sim 60 \mathrm{~nm}$ $\left(Q_{\text {Red,T }}=2.8 \mathrm{mC} \mathrm{cm}^{-2}\right)$ by using a reduction charge versus ellipsometric thickness working curve. Then, these POAPcoated gold films were employed as working electrodes. A large-area gold grid was used as counter electrode. All the potentials reported in this work are referred to the SCE.

A series of 8 electrodes were prepared (see first column in Table 1) and each one of them was successively employed in an individual experiment similar to that described in [1113]. That is, each POAP film, after being equilibrated within the potential region $-0.2<E<0.5 \mathrm{~V}$ in the supporting electrolyte solution, was soaked in a $0.1 \mathrm{M} \mathrm{H}_{2} \mathrm{SO}_{4}+0.05 \mathrm{M}$ $\mathrm{Fe}_{2}\left(\mathrm{SO}_{4}\right)_{3}$ solution for different time periods (see second column in Table 1). Then, each one of these electrodes was extracted from the solution containing ferric ions and it was copiously washed with the supporting electrolyte solution, then the electrode was again transferred to the electrochemical cell containing the supporting electrolyte solution. The corresponding $j-E$ responses were recorded for each one of the 8 POAP films. In order to reactivate these deactivated POAP films, which had been initially treated with the ferric cation solution, they were treated with a $0.1 \mathrm{M} \mathrm{NH}_{4} \mathrm{OH}$ solution.

In CV measurements a PAR Model 173 potentiostat and a PAR Model 175 function generator were used. An X1-X2Y Hewlett-Packard Model 7046 B Plotter was used to record the $j-E$ responses.

In SR measurements, the POAP-coated gold film conductivity was measured by circulating a $d c$ current $(1 \mathrm{~mA})$ between the extreme contacts while polarizing the electrode

Table 1. Voltammetric Reduction Charge $Q_{\text {Red,c }}$ and Degree of Deactivation, $\theta_{\mathrm{c}}^{\mathrm{d}}$, of Different POAP Films

\begin{tabular}{|c|c|c|c|}
\hline POAP films $^{\text {a }}$ & Soaking time $^{\mathbf{b}} / \mathbf{h}$ & $\boldsymbol{Q}_{\text {Red,c }}{ }^{\mathrm{c}} / \mathbf{m C c m}^{-2}$ & $\boldsymbol{\theta}_{\mathbf{c}}{ }^{\mathbf{d}}$ \\
\hline \hline 1 & 2 & 2.44 & 0.13 \\
\hline 2 & 4 & 2.27 & 0.19 \\
\hline 3 & 5 & 1.87 & 0.33 \\
\hline 4 & 10 & 1.68 & 0.40 \\
\hline 5 & 12 & 1.62 & 0.42 \\
\hline 6 & 16 & 1.54 & 0.45 \\
\hline 7 & 19 & 1.46 & 0.48 \\
\hline 8 & 24 & 1.34 & 0.52 \\
\hline
\end{tabular}

${ }^{\mathrm{a}}$ Numbers 1 to 8 represent different deactivated POAP films.

${ }^{\mathrm{b}}$ Different soaking times in a $0.1 \mathrm{M} \mathrm{H}_{2} \mathrm{SO}_{4}+0.05 \mathrm{M} \mathrm{Fe}_{2}\left(\mathrm{SO}_{4}\right)_{3}$ solution.

${ }^{c}$ Voltammetric reduction charge of the different deactivated POAP films after being subjected to the soaking process in the presence of the ferric cation solution.

${ }^{\mathrm{d}}$ Degree of deactivation of the different POAP films calculated from $\theta \mathrm{c}=1-\left(Q_{\text {Red, } \mathrm{C}} / Q_{\text {Red,T }}\right) . Q_{\text {Red,T }}\left(=2.8 \mathrm{mC} \mathrm{cm}^{-2}\right)$ is the voltammetric reduction charge of a non-deactivated film. 
ensemble through the central contact [14]. The resistance change obtained in this way was recorded as a function of the potential $(\Delta R / R-E)$, together with the voltammetric response $(j-E)$. The experimental setup for simultaneous voltammetric and SR measurements on thin film electrodes has previously been described in detail [14].

AR grade chemicals were used throughout. $O$ aminophenol (Fluka) was purified as described elsewhere $[5,6] . \mathrm{HClO}_{4}$ and $\mathrm{NaClO}_{4}$ (Merck) were used without further purification. Ferric sulphate and ammonium hydroxide (Mallinckrodt, analytical reagent grade) were employed in preparing the corresponding solutions. The solutions were prepared with water purified using a Millipore Milli-Q system.

\section{RESULTS AND DISCUSSION}

Fig. (1) shows simultaneous voltammetric and resistometric responses corresponding to a freshly prepared POAP film (non-deactivated). Figs. (2 and 3 ) show (j-E) and $(\Delta R / R-E)$ responses, respectively, of the films (1), (3) and (7) (see also Table 1) after being subjected to the soaking process previously described. It is not the aim of this work to add information about the process of interaction of iron cations with POAP films, but simply to employ the interaction effects to deactivate POAP films and then study the resistance response of deactivated films. However, electrochemical and spectroscopic experiments similar to those described in [13] were performed in the present work to verify the incorporation of iron ions in our POAP films. Our results are in complete agreement with those reported in [13]. Then, we conclude that the attenuated response of our POAP films, after their immersion in a ferric cation solution (Figs. $\mathbf{2}$ and 3), is indicative of the presence of iron ions inside the polymer matrix.

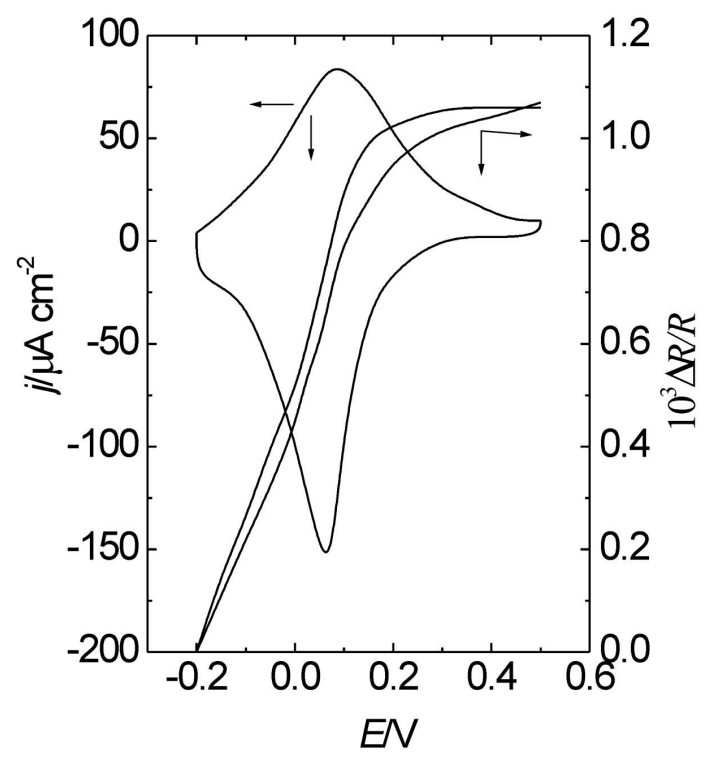

Fig. (1). Simultaneous $\Delta R / R-E$ and $j-E$ responses of a $30 \mathrm{~nm}$ thick gold film coated with a $2.8 \mathrm{mC} \mathrm{cm}^{-2}\left(\phi_{\mathrm{p}}=60 \mathrm{~nm}\right)$ thick POAP film. Electrolyte: $0.1 \mathrm{M} \mathrm{HClO}_{4}+0.4 \mathrm{M} \mathrm{NaClO}_{4}$. Scan rate: $v=0.01 \mathrm{~V} \mathrm{~s}^{-1}$.

The more attenuated voltammetric response of a POAP film with increasing soaking time in the ferric cation solution (Fig. 2) was employed to quantify the degree of deactivation of the polymer film. In this regard, voltammetric reduction charge values corresponding to the completely reduced POAP films were compared for both non-deactivated films $\left(Q_{\text {Red,T }}=2.8 \mathrm{mC} \mathrm{cm}^{-2}\right)$ and films subjected to the soaking process in the presence of the $\mathrm{Fe}(\mathrm{III})$ solution $\left(Q_{\text {Red,c }}\right)$ (see column 3 in Table 1); then, a degree of deactivation (column 4 of Table 1) was defined as

$\theta_{\mathrm{c}}=1-\left(Q_{\text {Red,c }} / Q_{\text {Red }, \mathrm{T}}\right)$

where $Q_{\text {Red,c }}$ is the total reduction charge assessed by integration of the corresponding $j-E$ response from $E=0.5 \mathrm{~V}$ towards the negative potential direction for a deactivated film and $Q_{\text {Red,T }}=2.8 \mathrm{mC} \mathrm{cm}^{-2}$ is the total reduction charge for the non-deactivated film. In this way, for a non-deactivated POAP film (dashed line in Fig. 2) the degree of deactivation was $\theta_{\mathrm{c}}=0$, taking $Q_{\mathrm{Red}, \mathrm{T}}=2.8 \mathrm{mC} \mathrm{cm}^{-2}$ as reference charge. However, values of $\theta_{c}>0$ are indicative of POAP films that have been deactivated.

With regard to SR measurements, an attenuation of the relative resistance change $(\Delta R / R-E)$ in going from the oxidized to the reduced state of POAP is also observed with the increase of the degree of deactivation. In this last response the potential value $E=0.5 \mathrm{~V}$ was taken as the reference to assess the resistance changes (Fig. 3). As can be seen from Fig. (3), the $\Delta R / R$ value at the reduced state $(E=-0.2 \mathrm{~V})$ for a deactivated POAP film is higher than that corresponding to the non-deactivated film. That is, the more deactivated the polymer film, the more attenuated is the resistance response in going from the oxidized to the reduced state.

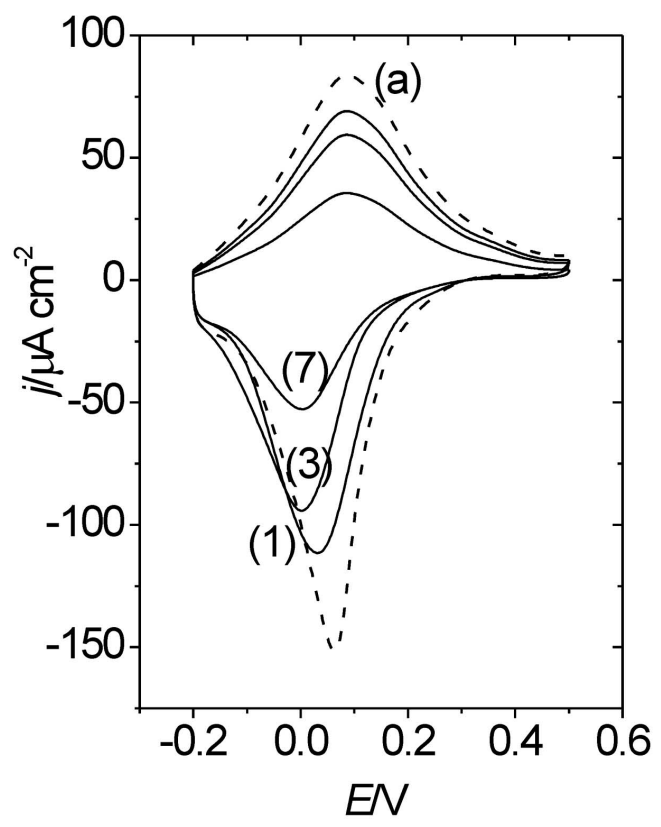

Fig. (2). Voltammetric $(j-E)$ responses for a $2.8 \mathrm{mC} \mathrm{cm}^{-2}\left(\phi_{\mathrm{p}}=60\right.$ $\mathrm{nm}$ ) thick POAP film. (a) a freshly prepared POAP film. Numbers (1), (3) and (7) correspond to POAP films with different degrees of deactivation (see Table 1). Electrolyte: $0.1 \mathrm{M} \mathrm{HClO}_{4}+0.4 \mathrm{M} \mathrm{Na}-$ $\mathrm{ClO}_{4}$. Scan rate: $v=0.01 \mathrm{~V} \mathrm{~s}^{-1}$.

As was above indicated, another experiment was performed with POAP films that, after being deactivated by treatment with the ferric cation solution, were treated with an ammonium solution, as reported in [13]. In this regard, Fig. (4) compares the voltammetric response of a non-deactivated film with those of the same film, firstly deactivated by soak- 


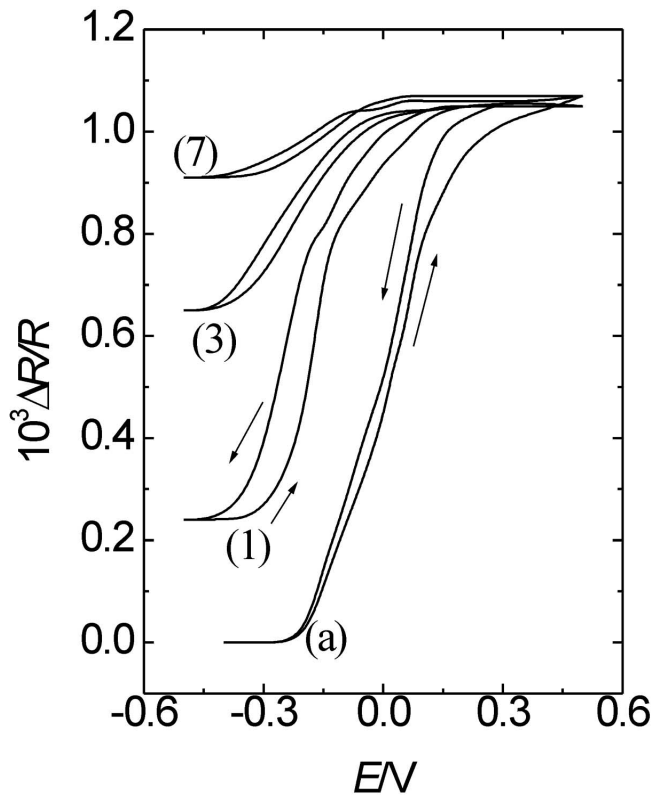

Fig. (3). $\Delta R / R-E$ responses obtained simultaneously with the (j-E) responses shown in Fig. (2). The same notation and conditions indicated in Fig. (2).

ing in a ferric cation solution and then reactivated by treatment with a $0.1 \mathrm{M} \mathrm{NH}_{4} \mathrm{OH}$ solution. As reported in [13], an enhancement of the redox response is observed after reactivation. Fig. (5) compares the $\Delta R / R-E$ dependence for the non-deactivated film (plot (a)) with those of the same film firstly deactivated (plot (b)) and then, reactivated (plot (c)) by alkaline treatment. As can be seen from Fig. (5) also, an enhancement of the resistance response is obtained with reactivation.

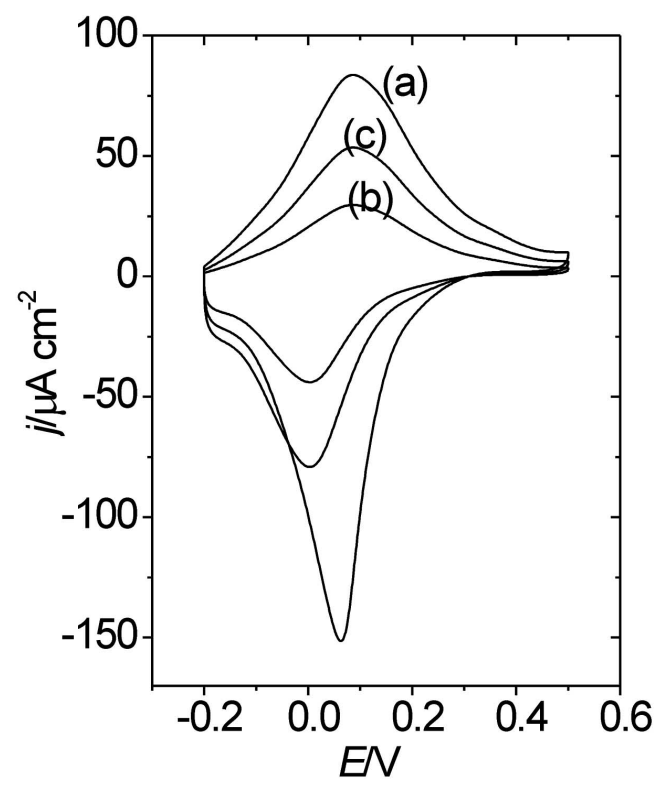

Fig. (4). Voltammetric (j-E) responses of film (3) (see Table 1): (a) Non-deactivated film, $\theta_{\mathrm{c}}=0$; (b) the same film deactivated, $\theta_{\mathrm{c}}=$ 0.33 ; (c) the same film after reactivation, $\theta_{\mathrm{c}}=0.15$. Electrolyte: 0.1 $\mathrm{M} \mathrm{HClO}_{4}+0.4 \mathrm{M} \mathrm{NaClO}_{4}$. Scan rate: $v=0.01 \mathrm{~V} \mathrm{~s}^{-1}$.

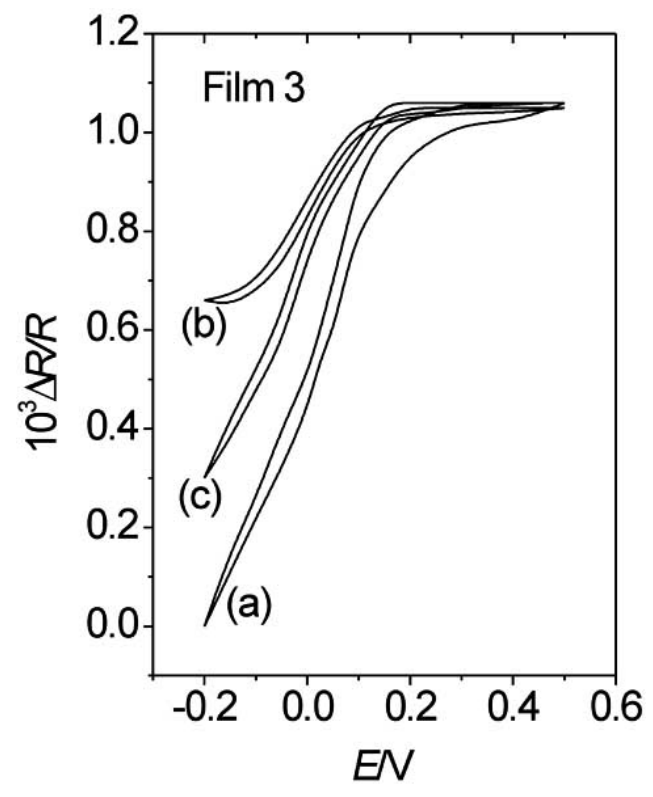

Fig. (5). $\Delta R / R-E$ responses obtained simultaneously with the $(j-E)$ responses shown in Fig. (4). The same notation and conditions indicated in Fig. (4).

\subsection{Interpretation of Surface Resistance Measurements}

Interpretation of SR measurements in Electrochemistry can be found in [14]. The resistance changes at the goldPOAP interface should not be considered as the direct result of the electron transfer between the polymeric molecules on the metal film surface and the metal, but rather they originate from the transformation of a given polymeric species in other on the metal film surface during the redox conversion of the polymer film [14]. These different species should act as different scattering centers on the metal film surface for the conduction electrons of the metal. Also, in terms of surface configurations of scatterers, it should be expected that compact distributions of species on the metal film reflect conduction electrons more specularly than expanded ones [14]. In the case of POAP deposited on gold films, the interconversion of amine to imine sites on the gold film surface during the redox process of POAP was considered as responsible for the observed resistometric changes of the gold films [14]. The development of a new distribution of scatterers at the gold-POAP interface during the redox conversion of POAP should reflect conduction electrons from the inside of the gold film on its surface with a different degree of specularity than the initial one. In this sense, taking as reference the oxidized state of POAP, the $\Delta R / R$ decrease in going towards the negative potential direction (plot (a) in Fig. 3), was attributed to the appearance of a new surface configuration of scatterers (amino species) during POAP reduction with a spacing among them constant and lower than that among imine sites in the oxidized state. In this way, the distribution of amine sites reflects conduction electrons more specularly than the distribution of imine sites. Consequently, the distribution of imine species in the oxidized state of POAP is less compact than the corresponding distribution of amine species in the reduced state, leading in this way to a more diffuse reflection of the conduction electrons in the oxidized state than in the reduced one. In this way, a higher $\Delta R / R$ va- 
lue is observed for the oxidized state of POAP as compared with the reduced one. A confirmation that supports a more extended configuration of oxidized sites, as compared with that of reduced ones, at POAP can be given in terms of gaps, which appear during POAP oxidation. In this regard, optical measurements on POAP films reveal that only one in every four or five amine sites is converted to the corresponding imine site [8]. Thus, the existence of inactive gaps within the distribution of oxidized sites of POAP could justify that POAP in its oxidized state reflect conduction electrons of gold more diffusely than in its reduced state. Further confirmation about the different reflecting properties of the oxidized and reduced states of POAP can be found in the different values of the site interaction parameters $(r)$ obtained from cathodic and anodic voltammetric response of POAP. Nonideal behavior of POAP, which is actually expected considering the rather high concentration of active sites in the film (i.e. $c=4.7 \mathrm{M}[6]$ ), leads to the following values of anodic and cathodic site interaction parameters: $r_{\mathrm{a}}=-0.55 \mathrm{M}^{-1}$ and $r_{\mathrm{c}}=-0.18 \mathrm{M}^{-1}$, respectively. Both are negative, thus involving a repulsive energy of interaction. As a higher repulsion is observed between oxidized sites than reduced ones at POAP, then a more extended configuration of oxidized sites should be expected as compared with the corresponding distribution of reduced sites. Then, it should be expected that oxidized sites reflect electrons more diffusely than the distribution of reduced sites.

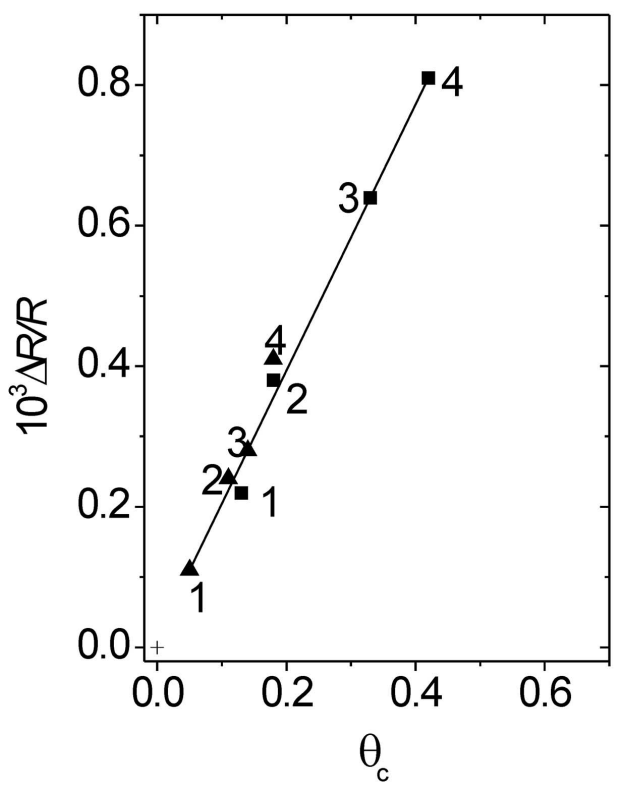

Fig. (6). $\Delta R / R$ values at $E=-0.2 \mathrm{~V} v s \theta_{\mathrm{c}}$ for film (1) to film (4) (see Table 1). ( $\square$ ) values corresponding to deactivated films; ( $\boldsymbol{\Delta})$ values corresponding to reactivated films. Electrolyte: $0.1 \mathrm{M} \mathrm{HClO}_{4}+0.4$ $\mathrm{M} \mathrm{NaClO}_{4}$.

As can be seen from Fig. (3), an attenuated resistance decrease (plots (1), (3) and (7)) is observed for deactivated POAP films in going from the oxidized to the reduced state, as compared with a non-deactivated film (plot (a) in Fig. 3). Also the higher is the $\theta_{\mathrm{c}}$ value the more attenuated becomes the resistance decrease. The attenuated resistance responses of deactivated POAP films, as compared with that exhibited by a film free of ferric cations, can be attributed to decrea- sing amounts of active amine redox sites in the reduced state of POAP. As was previously demonstrated by spectroelectrochemical measurements, the incorporation of ferric cations into POAP films affects the protonation reaction of the polymer [11]. That is, deactivation of POAP after contact with a ferric cation solution yields the inhibition of its protonation reaction. In terms of distribution of scatterers at the polymer-metal interface [14], the more attenuated resistance response of a POAP film with increasing its degree of deactivation (increase of the $\Delta R / R$ value at $E=-0.2 \mathrm{~V}$ ) can be attributed to the progressive appearance of more extended distributions of amine sites (higher distance between them) due to the creation of inactive gaps in the redox sites configuration. Then, the more extended distribution of amine sites in a deactivated POAP film, as compared with that of a nondeactivated one, leads to a more diffuse reflection of the conduction electrons of the gold film, and consequently to a higher $\Delta R / R$ value at $E=-0.2 \mathrm{~V}$.

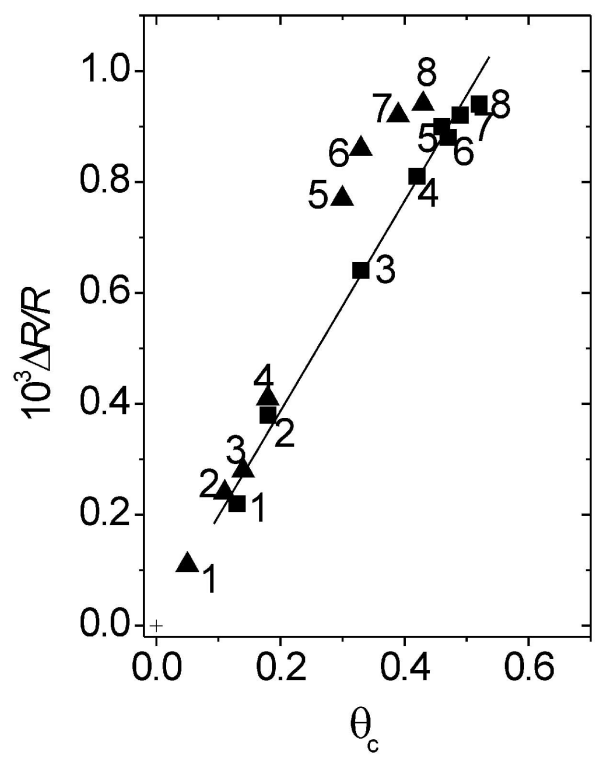

Fig. (7). $\Delta R / R$ values at $E=-0.2 \mathrm{~V} v s \theta_{\mathrm{c}}$ for film (1) to film (8) (see Table 1). ( $\square)$ values corresponding to deactivated films; $(\boldsymbol{\Delta})$ values corresponding to reactivated films. Electrolyte: $0.1 \mathrm{M} \mathrm{HClO}_{4}+0.4$ $\mathrm{M} \mathrm{NaClO}_{4}$.

As was above indicated, deactivated POAP films can be partially reactivated (Figs. $\mathbf{4}$ and $\mathbf{5}$ ) by treatment with an ammonium solution, which eliminates part of ferric cations incorporated into the POAP matrix [13]. The $\Delta R / R$ value at the reduced state of POAP $(E=-0.2)$ for each one of the deactivated and then, reactivated films, was represented as a function of $\theta_{\mathrm{c}}$ (Figs. 6 and 7). As can be seen from Fig. (6), up to a $\theta_{\mathrm{c}}$ value around 0.40 , a linear $\Delta R / R v s$. $\theta_{\mathrm{c}}$ dependency is observed (slope $\Delta R / R \theta_{\mathrm{c}}=2$ ). In this regard, reactivation of POAP films with low degrees of deactivation $\left(\theta_{c}<0.40\right)$ allows one not only to recover part of the active redox sites but also to maintain constant their distributions [14]. However, even when for deactivated films with higher $\theta_{\mathrm{c}}$ values (> 0.40 , films 5 to 8$) \Delta R / R$ as a function of $\theta_{\mathrm{c}}$ follows the same linear dependency as for lower $\theta_{\mathrm{c}}$ values $(<0.40), \Delta R / R$ values for the reactivated films are placed above the linear dependence (Fig. 7). On the basis of SR measurements, this last effect can be associated to a lack of relaxation of the redox sites configuration at the POAP-metal interface, after 
to reduce the amount of ferric cations contained into the film (reactivation). That is, even when it is possible to reactivate, strongly deactivated POAP films $\left(\theta_{\mathrm{c}}>0.4\right)$, the distribution of redox sites (distances between active redox sites) does not remain constant after reactivation. This fact should be indicative of a permanent distortion of the redox sites configuration of POAP after a strong deactivation by interaction with ferric cations. In this regard, besides the creation of inactive gaps (inhibition of the protonation reaction) during the incorporation of ferric cations into the POAP matrix, there is another effect that can also contribute to the existence of extended redox sites configurations. This effect is related to the existence of a strong repulsive interaction between positive charged sites within the polymer matrix due to incorporation of large amounts of $\mathrm{Fe}(\mathrm{III})$ cations. Large amounts of ferric cations $\left(\theta_{\mathrm{c}}>0.40\right)$ incorporated into POAP should cause that charged groups tend to become apart from each other, thus extending the redox sites configuration. However, it is possible that even when part of a large amount of accumulated positive charge was eliminated by reactivation, the original distribution of redox sites is not totally recovered, that is, a permanent change in the distance between active redox sites should remain. As electron hopping controls the charge-transport process at POAP [4], it is evident that an increase in the distance between adjacent redox sites should reduce the electron transport rate. In this regard, in a previous work [12] was demonstrated that the efficiency of POAP to act as redox mediator is strongly reduced after its interaction with a ferric cation solution.

\section{CONCLUSIONS}

Deactivation of POAP films after treatment with a ferric cation solution, was studied by Surface Resistance measurements. Resistance changes at the reduced state of POAP, allowed one to infer that inactive zones are developed within the redox sites configuration of a deactivated film. Inactive zones were attributed to the inhibition of the protonation reaction by the ferric cations captured by POAP. Deactivated POAP films can be reactivated by alkaline treatment. However, the distance between redox sites after reactivation depends on the initial degree of deactivation. In this regard, POAP films with a low degree of deactivation $\left(\theta_{c}<0.40\right)$ were reactivated without distortion of their redox sites configuration. However, a more expanded redox sites distribution were observed after reactivation of a strongly deactivated film $\left(\theta_{c}>0.40\right)$. The latter effect was attributed to strong repulsive interactions between charged sites, which should operate when large amounts of positive charges are incorporated into POAP.

\section{ACKNOWLEDGEMENTS}

The authors gratefully acknowledge the Consejo Nacional de Investigaciones Científicas y Técnicas (CONICET) and also the Facultad de Ciencias Exactas, National University of La Plata (UNLP).

\section{REFERENCES}

[1] Inzelt, G.; Pineri, M.; Schultze, J.W.; Vorotyntsev, M.A. Electron and proton conducting polymers: recent developments and prospects, Electrochim. Acta, 2000, 45, 2403-2421.

[2] Anderson, L.J.; Coury Jr., L.A.; Leddy, J. Dynamic electrochemistry: Methodol. Appl. Anal. Chem., 1998, 7, 519R-589R.

[3] Malev, V.V.; Kondratiev, V.V. Charge transfer processes in conducting polymer films. Russ. Chem. Rev., 2006, 75, 147-160.

[4] Tucceri, R. A review about the charge conduction process at poly(o-aminophenol) film electrodes. Open Phys. Chem. J., 2009, $3,30-45$.

[5] Barbero, C.; Silber, J.J.; Sereno, L. Formation of a novel electroactive film by electropolymerization of orthoaminophenol. Study of its chemical structure and formation mechanism. Electropolymerization of analogous compounds. J. Electroanal. Chem., 1989, 263, 333-352.

[6] Barbero, C.; Silber, J. J.; Sereno, L. Electrochemical properties of poly-ortho-aminophenol modified electrodes in aqueous acid solutions. J. Electroanal. Chem., 1990, 291, 81.

[7] Ohsaka, T.; Kunimura, S.; Oyama, N. Electrode kinetics of poly $(o$ aminophenol) film prepared by electrooxidative polymerization of aminophenol and its electrochromic propertes. Electrochim. Acta, 1988, 33, 639-645.

[8] Tucceri, R.I.; Barbero, C.; Silber, J.J.; Sereno, L.; Posadas, D. Spectroelectrochemical study of poly(o-aminophenol). Electrochim. Acta, 1997, 42, 919-927.

[9] Bonfranceschi, A.; Pérez Córdoba, A.; Keunchkarian, S.; Zapata, S.; Tucceri, R. Transport across poly(o-aminophenol) modified electrodes in contact with media containing redox active couples. A study using rotating disc electrode voltammetry. J. Electroanal. Chem., 1999, 477, 1 .

[10] Tucceri, R. Practical applications of poly $(o$-aminophenol) film electrodes. Open Phys. Chem. J., 2009, 3, 46-62.

[11] Scolari, D.; Tucceri, R. Deactivation of poly(o-aminophenol) after its interaction with ferric cations. Effects on the protonation reaction and electron transport. Open Phys. Chem. J., 2009, 3, 79-84.

[12] Tucceri, R. Redox mediation and permeation processes at deactivated poly $(o$-aminophenol) films. A study applying rotating disc electrode voltammetry and electrochemical impedance spectroscopy. J. Electroanal. Chem., 2009, 633, 198-206.

[13] Yano, J.; Kawakami, H.; Yamasaki, S.; Kanno, Y. Cation capturing ability and the potential response of a poly(o-aminophenol) film electrode to dissolved ferric ions. J. Electrochem. Soc., 2001, 148, E61-E65.

[14] Tucceri, R. A review about the surface resistance technique in electrochemistry. Surf. Sci. Rep., 2004, 56, 85-157.

[15] Barbero, C.; Zerbino, J.; Sereno, L.; Posadas, D. Optical properties of electropolymerized orthoaminophenol. Electrochim. Acta, 1987, 32, 693-697.

Received: January 22, 2010

(C) Scolari and Tucceri; Licensee Bentham Open.

This is an open access article licensed under the terms of the Creative Commons Attribution Non-Commercial License (http://creativecommons.org/licenses/by-nc/3.0/) which permits unrestricted, non-commercial use, distribution and reproduction in any medium, provided the work is properly cited. 